\title{
Spectra of the Energy Operator of Four-Electron Systems in the Impurity Hubbard Model. Triplet State
}

\author{
S. M. Tashpulatov, R. T. Parmanova \\ Institute of Nuclear Physics of Academy of Science of Republic of Uzbekistan, Tashkent, Uzbekistan \\ Email: sadullatashpulatov@yandex.ru, toshpul@mail.ru, toshpul@inp.uz
}

How to cite this paper: Tashpulatov, S.M. and Parmanova, R.T. (2021) Spectra of the Energy Operator of Four-Electron Systems in the Impurity Hubbard Model. Triplet State. Journal of Applied Mathematics and Physics, 9, 2776-2795.

https://doi.org/10.4236/jamp.2021.911179

Received: September 18, 2021

Accepted: November 14, 2021

Published: November 17, 2021

Copyright (c) 2021 by author(s) and Scientific Research Publishing Inc. This work is licensed under the Creative Commons Attribution International License (CC BY 4.0).

http://creativecommons.org/licenses/by/4.0/

\begin{abstract}
We consider the energy operator of four-electron systems in an impurity Hubbard model and investigated the structure of essential spectra and discrete spectrum of the system in the first triplet state in a one-dimensional lattice. For investigation the structure of essential spectra and discrete spectrum of the energy operator of four-electron systems in an impurity Hubbard model, for which the momentum representation is convenient. In addition, we used the tensor products of Hilbert spaces and tensor products of operators in Hilbert spaces and described the structure of essential spectrum and discrete spectrum of the energy operator of four-electron systems in an impurity Hubbard model. The investigations show that there are such cases: 1) the essential spectrum of the system consists of the union of no more than eight segments, and the discrete spectrum of the system consists of no more than three eigenvalues; 2) the essential spectrum of the system consists of the union of no more than sixteen segments, and the discrete spectrum of the system consists of no more than eleven eigenvalues; 3 ) the essential spectrum of the system consists of the union of no more than three segments, and the discrete spectrum of the system is the empty set. Consequently, the essential spectrum of the system consists of the union of no more than sixteen segments, and the discrete spectrum of the system consists of no more than eleven eigenvalues.
\end{abstract}

\section{Keywords}

Hubbard Model, Essential Spectrum, Discrete Spectrum, Four Electron Systems, Quintet State, Triplet State, Singlet State, Spectra

\section{Introduction}

In the early 1970s, three papers [1] [2] [3], where a simple model of metal was 
proposed that has become a fundamental model in the theory of strongly correlated electron systems, appeared almost simultaneously and independently. In that model, a single nondegenerate electron band with a local Coulomb interaction is considered. The model Hamiltonian contains only two parameters: the matrix element $t$ of electron hopping from a lattice site to a neighboring site and the parameter $U$ of the on-site Coulomb repulsion of two electrons. In the secondary quantization representation, the Hamiltonian can be written as

$$
H=t \sum_{m, \gamma} a_{m, \gamma}^{+} a_{m, \gamma}+U \sum_{m} a_{m, \uparrow}^{+} a_{m, \uparrow} a_{m, \downarrow}^{+} a_{m, \downarrow},
$$

where $a_{m, \gamma}^{+}$and $a_{m, \gamma}$ denote Fermi operators of creation and annihilation of an electron with spin $\gamma$ on a site $m$ and the summation over $\tau$ means summation over the nearest neighbors on the lattice.

The model proposed in [1] [2] [3] was called the Hubbard model after John Hubbard, who made a fundamental contribution to studying the statistical mechanics of that system, although the local form of Coulomb interaction was first introduced for an impurity model in metal by Anderson [4]. We also recall that the Hubbard model is a particular case of the Shubin-Wonsowsky polaron model [5], which had appeared 30 years before [1] [2] [3]. In the Shubin-Wonsowsky model, along with the on-site Coulomb interaction, the interaction of electrons on neighboring sites is also taken into account.

The Hubbard model is currently one of the most extensively studied multielectron models of metals [6] [7] [8] [9] [10]. Therefore, obtaining exact results for the spectrum and wave functions of the crystal described by the Hubbard model is of great interest. The spectrum and wave functions of the system of two electrons in a crystal described by the Hubbard Hamiltonian were studied in [6]. It is known that two-electron systems can be in two states, triplet and singlet [6] [7] [8] [9] [10]. It was proved in [6] that the spectrum of the system Hamiltonian $H^{t}$ in the triplet state is purely continuous and coincides with a segment $[m, M]=[2 A-4 B v, 2 A+4 B v]$, and the operator $H^{s}$ of the system in the singlet state, in addition to the continuous spectrum $[m, M]$, has a unique antibound state for some values of the quasimomentum.

The spectrum and wave functions of the system of three electrons in a crystal described by the Hubbard Hamiltonian were studied in [11]. The three-electron systems exist quartet state, and two type doublet states.

The spectrum of the energy operator of a system of four electrons in a crystal described by the Hubbard Hamiltonian in the triplet state was studied in [12]. The four-electron systems exist quintet states, and three type triplet states, and two type singlet states. The triplet state corresponds to the basic functions ${ }^{1} t_{m, n, p, r}^{1}=a_{m, \uparrow}^{+} a_{n, \uparrow}^{+} a_{p, \uparrow}^{+} a_{r, \downarrow}^{+} \varphi_{0},{ }^{2} t_{m, n, p, r}^{1}=a_{m, \uparrow}^{+} a_{n, \uparrow}^{+} a_{p, \downarrow}^{+} a_{r, \uparrow}^{+} \varphi_{0}$, ${ }^{3} t_{m, n, p, r}^{1}=a_{m, \uparrow}^{+} a_{n, \downarrow}^{+} a_{p, \uparrow}^{+} a_{r, \uparrow}^{+} \varphi_{0}$.

The spectrum of the energy operator of four-electron systems in the Hubbard model in the quintet and singlet states was studied in [13]. The quintet state corresponds to the free motion of four electrons over the lattice with the basic 
functions $q_{m, n, p, r}^{2}=a_{m, \uparrow}^{+} a_{n, \uparrow}^{+} a_{p, \uparrow}^{+} a_{r, \uparrow}^{+} \varphi_{0}$. The work [13] proved, that the spectrum of the system in a quintet state is purely continuous and coincides with the segment $[4 A-8 B v, 4 A+8 B v]$, and the four-electron bound states or the four-electron antibound states are absent. The singlet state corresponds to the basic functions ${ }^{1} s_{p, q, r, t}^{0}=a_{p, \uparrow}^{+} a_{q, \uparrow}^{+} a_{r, \downarrow}^{+} a_{t, \downarrow}^{+} \varphi_{0},{ }^{2} s_{p, q, r, t}^{0}=a_{p, \uparrow}^{+} a_{q, \downarrow}^{+} a_{r, \uparrow}^{+} a_{t, \downarrow}^{+} \varphi_{0}$, and these two singlet states have different origins.

The use of films in various areas of physics and technology arouses great interest in studying a localized impurity state (LIS) of a magnet. Therefore, it is important to study the spectral properties of electron systems in the impurity Hubbard model. The spectrum of the energy operator of three-electron systems in the Impurity Hubbard model in the second doublet state was studied [14]. The structure of essential spectra and discrete spectrum of three-electron systems in the impurity Hubbard model in the Quartet state were studied in [15].

\section{Hamiltonian of the System}

We consider the energy operator of four-electron systems in the Impurity Hubbard model and describe the structure of the essential spectra and discrete spectrum of the system for the first triplet states in the one-dimensional lattice. The Hamiltonian of the chosen model has the form

$$
\begin{aligned}
H= & A \sum_{m, \gamma} a_{m, \gamma}^{+} a_{m, \gamma}+B \sum_{m, \tau, \gamma} a_{m, \gamma}^{+} a_{m+\tau, \gamma}+U \sum_{m} a_{m, \uparrow}^{+} a_{m, \uparrow} a_{m, \downarrow}^{+} a_{m, \downarrow} \\
& +\left(A_{0}-A\right) \sum_{\gamma} a_{0, \gamma}^{+} a_{0, \gamma}+\left(B_{0}-B\right) \sum_{\tau, \gamma}\left(a_{0, \gamma}^{+} a_{\tau, \gamma}+a_{\tau, \gamma}^{+} a_{0, \gamma}\right) \\
& +\left(U_{0}-U\right) a_{0, \uparrow}^{+} a_{0, \uparrow} a_{0, \downarrow}^{+} a_{0, \downarrow} .
\end{aligned}
$$

Here $A\left(A_{0}\right)$ is the electron energy at a regular (impurity) lattice site, $B\left(B_{0}\right)$ is the transfer integral between (between electron and impurities) neighboring sites (we assume that $B>0\left(B_{0}>0\right)$ for convenience), $\tau= \pm e_{j}, j=1,2, \cdots, v$, where $e_{j}$ are unit mutually orthogonal vectors, which means that summation is taken over the nearest neighbors, $U\left(U_{0}\right)$ is the parameter of the on-site Coulomb interaction of two electrons in the regular (impurity) sites, $\gamma$ is the spin index, $\gamma=\uparrow$ or $\gamma=\downarrow, \uparrow$ and $\downarrow$ denote the spin values $\frac{1}{2}$ and $-\frac{1}{2}$, and $a_{m, \gamma}^{+}$and $a_{m, \gamma}$ are the respective electron creation and annihilation operators at a site $m \in Z^{v}$.

The four-electron systems have a quintet state, and two type singlet state, and three type triplet states. The energy of the system depends on its total spin $S$. Along with the Hamiltonian, the $N_{e}$ electron system is characterized by the total spin $S, S=S_{\text {max }}, S_{\text {max }}-1, \cdots, S_{\text {min }}, S_{\text {max }}=\frac{N_{e}}{2}, S_{\text {min }}=0, \frac{1}{2}$.

Hamiltonian (2) commutes with all components of the total spin operator $S=\left(S^{+}, S^{-}, S^{z}\right)$, and the structure of eigenfunctions and eigenvalues of the system, therefore, depends on $S$. The Hamiltonian $H$ acts in the antisymmetric Fock space $\mathcal{H}_{a s}$. 


\section{Four-Electron First Triplet State in the Impurity Hubbard Model}

Let $\varphi_{0}$ be the vacuum vector in the space $\mathcal{H}_{a s}$. The first triplet state corresponds to the free motion of four electrons and their interactions over the lattice with the basic functions ${ }^{1} t_{p, q, r, k \in Z^{\nu}}^{1}=a_{p, \uparrow}^{+} a_{q, \uparrow}^{+} a_{r, \uparrow}^{+} a_{k, \downarrow}^{+} \varphi_{0}$. The subspace ${ }^{1} \mathcal{H}_{1}^{t}$, corresponding to the first triplet state is the set of all vectors of the form ${ }^{1} \psi_{1}^{t}=\sum_{p, q, r, k \in Z^{v}} f(p, q, r, k){ }^{1} t_{p, q, r, k \in Z^{\nu}}^{1}, f \in l_{2}^{a s}$, where $l_{2}^{a s}$ is the subspace of antisymmetric functions in the space $l_{2}\left(\left(Z^{v}\right)^{4}\right)$. We denote by ${ }^{1} H_{1}^{t}$ the restriction of operator $H$ to the subspace ${ }^{1} \mathcal{H}_{1}^{t}$. We let $\varepsilon_{1}=A_{0}-A$, and $\varepsilon_{2}=B_{0}-B$, and $\varepsilon_{3}=U_{0}-U$.

Theorem 1. (coordinate representation of the action of operator ${ }^{1} H_{1}^{t}$ ) The subspace ${ }^{1} \mathcal{H}_{1}^{t}$ is invariant under the operator $H$, and the restriction ${ }^{1} H_{1}^{t}$ of operator $H$ to the subspace ${ }^{1} \mathcal{H}_{1}^{t}$ is a bounded self-adjoint operator. It generates a bounded self-adjoint operator ${ }^{1} \bar{H}_{1}^{t}$ acting in the space $l_{2}^{a s}$ as

$$
\begin{aligned}
{ }^{1} \bar{H}_{1}^{t 1} \psi_{1}^{t}= & 4 A f(p, q, r, k)+B \sum_{\tau}[f(p+\tau, q, r, k)+f(p, q+\tau, r, k) \\
& +f(p, q, r+\tau, k)+f(p, q, r, k+\tau)]+U\left[\delta_{p, k}+\delta_{q, k}+\delta_{r, k}\right] f(p, q, r, k) \\
& +\varepsilon_{1}\left[\delta_{p, 0}+\delta_{q, 0}+\delta_{r, 0}+\delta_{k, 0}\right] f(p, q, r, k)+\varepsilon_{2} \sum_{\tau}\left[\delta_{p, 0} f(\tau, q, r, k)\right. \\
& +\delta_{q, 0} f(p, \tau, r, k)+\delta_{r, 0} f(p, q, \tau, k)+\delta_{k, 0} f(p, q, r, \tau)+\delta_{p, \tau} f(0, q, r, k) \\
& \left.+\delta_{q, \tau} f(p, 0, r, k)+\delta_{r, \tau} f(p, q, 0, k)+\delta_{k, \tau} f(p, q, r, 0)\right] \\
& +\varepsilon_{3}\left[\delta_{p, 0} \delta_{k, 0}+\delta_{q, 0} \delta_{k, 0}+\delta_{r, 0} \delta_{k, 0}\right] f(p, q, r, k) .
\end{aligned}
$$

The operator ${ }^{1} H_{1}^{t}$ acts on a vector ${ }^{1} \psi_{1}^{t} \in{ }^{1} \mathcal{H}_{1}^{t}$ as

$$
{ }^{1} H_{1}^{t} \psi_{1}^{t}=\sum_{p, q, r, k \in Z^{\nu}}\left({ }^{1} \bar{H}_{1}^{t} f\right)(p, q, r, k){ }^{1} t_{p, q, r, k \in Z^{\nu}} .
$$

Proof. We act with the Hamiltonian $H$ on vectors ${ }^{1} \psi_{1}^{t} \in{ }^{1} \mathcal{H}_{1}^{t}$ using the standard anticommutation relations between electron creation and annihilation operators at lattice sites, $\left\{a_{m, \gamma}, a_{n, \beta}^{+}\right\}=\delta_{m, n} \delta_{\gamma, \beta},\left\{a_{m, \gamma}, a_{n, \beta}\right\}=\left\{a_{m, \gamma}^{+}, a_{n, \beta}^{+}\right\}=\theta$, and also take into account that $a_{m, \gamma} \varphi_{0}=\theta$, where $\theta$ is the zero element of ${ }^{1} \mathcal{H}_{1}^{t}$. This yields the statement of the theorem.

Lemma 1. The spectra of the operators ${ }^{1} H_{1}^{t}$ and ${ }^{1} \bar{H}_{1}^{t}$ coincide.

Proof. Because the operators ${ }^{1} H_{1}^{t}$ and ${ }^{1} \bar{H}_{1}^{t}$ are bounded self-adjoint operators, it follows that if $\lambda \in \sigma\left({ }^{1} H_{1}^{t}\right)$, then the Weyl criterion (see [16], chapter VII, paragraph 3, pp. 262-263) implies that there is a sequence $\left\{\psi_{n}\right\}_{n=1}^{\infty}$ such that $\left\|\psi_{n}\right\|=1$ and $\lim _{n \rightarrow \infty}\left\|\left({ }^{1} H_{1}^{t}-\lambda\right) \psi_{n}\right\|=0$. We set $\psi_{n}=\sum_{p, q, r, k} f_{n}(p, q, r, k) a_{p, \uparrow}^{+} a_{q, \uparrow}^{+} a_{r, \uparrow}^{+} a_{k, \downarrow}^{+} \varphi_{0}$. Then

$$
\begin{aligned}
& \left\|\left({ }^{1} H_{1}^{t}-\lambda\right) \psi_{n}\right\|^{2}=\left(\left({ }^{1} H_{1}^{t}-\lambda\right) \psi_{n},\left({ }^{1} H_{1}^{t}-\lambda\right) \psi_{n}\right) \\
& =\sum_{p, q, r, k}\left\|\left({ }^{1} \bar{H}_{1}^{t}-\lambda\right) f_{n}(p, q, r, k)\right\|^{2}\left(a_{p, \uparrow}^{+} a_{q, \uparrow}^{+} a_{r, \uparrow}^{+} a_{k, \downarrow}^{+} \varphi_{0}, a_{p, \uparrow}^{+} a_{q, \uparrow}^{+} a_{r, \uparrow}^{+} a_{k, \downarrow}^{+} \varphi_{0}\right) \\
& =\sum_{p, q, r, k}\left\|\left({ }^{1} \bar{H}_{1}^{t}-\lambda\right) F_{n}(p, q, r, k)\right\|^{2}\left(a_{k, \downarrow} a_{r, \uparrow} a_{q, \uparrow} a_{p, \uparrow} a_{p, \uparrow}^{+} a_{q, \uparrow}^{+} a_{r, \uparrow}^{+} a_{k, \downarrow}^{+} \varphi_{0}, \varphi_{0}\right)
\end{aligned}
$$




$$
\begin{aligned}
& =\sum_{p, q, r, k}\left\|\left({ }^{1} \bar{H}_{1}^{t}-\lambda\right) F_{n}(p, q, r, k)\right\|^{2}\left(\varphi_{0}, \varphi_{0}\right) \\
& =\sum_{p, q, r, k}\left\|\left({ }^{1} \bar{H}_{1}^{t}-\lambda\right) F_{n}(p, q, r, k)\right\|^{2} \rightarrow 0, \text { as } n \rightarrow \infty
\end{aligned}
$$

where $F_{n}=\sum_{p, q, r, k} f_{n}(p, q, r, k)$. It follows that $\lambda \in \sigma\left({ }^{1} \bar{H}_{1}^{t}\right)$. Consequently, $\sigma\left({ }^{1} H_{1}^{t}\right) \subset \sigma\left({ }^{1} \bar{H}_{1}^{t}\right)$.

Conversely, let $\bar{\lambda} \in \sigma\left({ }^{1} \bar{H}_{1}^{t}\right)$. Then, by the Weyl criterion, there is a sequence $\left\{F_{n}\right\}_{n=1}^{\infty}$ such that $\left\|F_{n}\right\|=1$ and $\lim _{n \rightarrow \infty}\left\|\left({ }^{1} \bar{H}_{1}^{t}-\bar{\lambda}\right) \psi_{n}\right\|=0$. Setting $F_{n}=\sum_{p, q, r, k} f_{n}(p, q, r, k),\left\|F_{n}\right\|=\left(\sum_{p, q, r, k}\left|f_{n}(p, q, r, k)\right|^{2}\right)^{\frac{1}{2}}$, we conclude that $\left\|\psi_{n}\right\|=\left\|F_{n}\right\|=1$ and $\left\|\left({ }^{1} \bar{H}_{1}^{t}-\bar{\lambda}\right) F_{n}\right\|=\left\|\left({ }^{1} \bar{H}_{1}^{t}-\bar{\lambda}\right) \psi_{n}\right\| \rightarrow 0$ as $n \rightarrow \infty$. This means that $\bar{\lambda} \in \sigma\left({ }^{1} H_{1}^{t}\right)$ and hence $\sigma\left({ }^{1} \bar{H}_{1}^{t}\right) \subset \sigma\left({ }^{1} H_{1}^{t}\right)$. These two relations imply $\sigma\left({ }^{1} H_{1}^{t}\right)=\sigma\left({ }^{1} \bar{H}_{1}^{t}\right)$.

We call the operator ${ }^{1} H_{1}^{t}$ the four-electron first triplet state operator in the impurity Hubbard model.

Let $\mathcal{F}: l_{2}\left(\left(Z^{v}\right)^{4}\right) \rightarrow L_{2}\left(\left(T^{v}\right)^{4}\right) \equiv{ }^{1} \tilde{H}_{1}^{t}$ be the Fourier transform, where $T^{v}$ is the $v$-dimensional torus endowed with the normalized Lebesgue measure d $\lambda$, i.e. $\lambda\left(T^{v}\right)=1$.

We set ${ }^{1} \tilde{H}_{1}^{t}=\mathcal{F}^{1} \bar{H}_{1}^{t} \mathcal{F}^{-1}$. In the quasimomentum representation, the operator ${ }^{1} \bar{H}_{1}^{t}$ acts in the Hilbert space $L_{2}^{a s}\left(\left(T^{v}\right)^{4}\right)$, where $L_{2}^{a s}$ is the subspace of antisymmetric functions in $L_{2}\left(\left(T^{v}\right)^{4}\right)$.

Theorem 2. (quasimomentum representation of the action of operator ${ }^{1} H_{1}^{t}$ ) The Fourier transform of operator ${ }^{1} \bar{H}_{1}^{t}$ is an operator ${ }^{1} \tilde{H}_{1}^{t}=\mathcal{F}^{1} \bar{H}_{1}^{t} \mathcal{F}^{-1}$ acting in the space $L_{2}^{a s}\left(\left(T^{v}\right)^{4}\right)$ be the formula

$$
\begin{aligned}
{ }^{1} \tilde{H}_{1}^{t 1} \psi_{1}^{t}= & h(\lambda, \mu, \gamma, \theta) f(\lambda, \mu, \gamma, \theta)+U\left[\int_{T^{v}} f(s, \mu, \gamma, \lambda+\theta-s) \mathrm{d} s\right. \\
& \left.+\int_{T^{v}} f(\lambda, u, \gamma, \mu+\theta-u) \mathrm{d} u+\int_{T^{v}} f(\lambda, \mu, v, \gamma+\theta-v) \mathrm{d} v\right] \\
& +\varepsilon_{1}\left[\int_{T^{v}} f(s, \mu, \gamma, \theta) \mathrm{d} s+\int_{T^{v}} f(\lambda, u, \gamma, \theta) \mathrm{d} u+\int_{T^{v}} f(\lambda, \mu, v, \theta) \mathrm{d} v\right. \\
& \left.+\int_{T^{v}} f(\lambda, \mu, \gamma, \xi) \mathrm{d} \xi\right]+2 \varepsilon_{2}\left[\int_{T^{v}} \sum_{i=1}^{v}\left[\cos \lambda_{i}+\cos s_{i}\right] f(s, \mu, \gamma, \theta) \mathrm{d} s\right. \\
& +\int_{T^{v}} \sum_{i=1}^{v}\left[\cos \mu_{i}+\cos u_{i}\right] f(\lambda, u, \gamma, \theta) \mathrm{d} u \\
& +\int_{T^{v}} \sum_{i=1}^{v}\left[\cos \gamma_{i}+\cos v_{i}\right] f(\lambda, \mu, v, \theta) \mathrm{d} v \\
& \left.+\int_{T^{v}} \sum_{i=1}^{v}\left[\cos \theta_{i}+\cos \xi_{i}\right] f(\lambda, \mu, \gamma, \xi) \mathrm{d} \xi\right] \\
& +\varepsilon_{3} \int_{T^{v}} \int_{T^{v}}[f(s, \mu, \gamma, \xi) \mathrm{d} s \mathrm{~d} \xi+f(\lambda, u, \gamma, \xi) \mathrm{d} u \mathrm{~d} \xi \\
& +f(\lambda, \mu, v, \xi) \mathrm{d} v \mathrm{~d} \xi],
\end{aligned}
$$

where $h(\lambda, \mu, \gamma, \theta)=4 A+2 B \sum_{i=1}^{v}\left[\cos \lambda_{i}+\cos \mu_{i}+\cos \gamma_{i}+\cos \theta_{i}\right]$, and $L_{2}^{a s}$ is the subspace of antisymmetric functions in $L_{2}\left(\left(T^{v}\right)^{4}\right)$.

Proof. The proof is by direct calculation in which we use the Fourier transformation in formula (3). 
In the impurity Hubbard model, the spectral properties of the considered operator of the energy of four-electron systems are closely related to those of its two-particle subsystems (one-electron systems with impurity). We first study the spectrum and localized impurity electron states of the one-electron impurity systems.

\section{One-Electron Systems in the Impurity Hubbard Model}

The Hamiltonian of one-electron systems in the impurity Hubbard model also has form (2). We let $\mathcal{H}_{1}$ denote the space of one-electron states of the operator $H$. It is clear that the space $\mathcal{H}_{1}$ is also invariant under operator $H$. We let $H_{1}$ denote the restriction of $H$ to the space $\tilde{\mathcal{H}}_{1}$.

Theorem 3. (coordination representation of the action of operator $H_{1}$ ) The space $\mathcal{H}_{1}$ is an invariant under operator $H$, and restriction $H_{1}$ of operator $H$ to the subspace $\mathcal{H}_{1}$ is a bounded self-adjoint operator. It generates a bounded self-adjoint operator $\bar{H}_{1}$, acting in the space $l_{2}^{a s}$ as

$$
\begin{aligned}
\left(\bar{H}_{1} f\right)(p)= & A f(p)+B \sum_{\tau}[f(p+\tau)]+\varepsilon_{1} \delta_{p, 0} f(p) \\
& +\varepsilon_{2} \sum_{\tau}\left[\delta_{p, 0} f(\tau)+\delta_{p, \tau} f(0)\right],
\end{aligned}
$$

where $\delta_{k, j}$ is the Kronecker symbol. The operator $H_{1}$ acts on a vector $\psi \in \mathcal{H}_{1}$ as

$$
H_{1}=\sum_{p, \tau}\left(\bar{H}_{1} f\right)(p) a_{p, \uparrow}^{+} \varphi_{0} .
$$

Lemma 2. The spectra of the operators $H_{1}$ and $\bar{H}_{1}$ coincide.

We let $\mathcal{F}$ denote the Fourier transform: $\mathcal{F}: l_{2}\left(Z^{v}\right) \rightarrow L_{2}\left(T^{v}\right)$. We set $\tilde{H}_{1}=\mathcal{F} \bar{H}_{1} \mathcal{F}^{-1}$. In the quasimomentum representation, the operator $\bar{H}_{1}$ acts in the Hilbert space $L_{2}\left(T^{v}\right)$.

Theorem 4. (quasimomentum representation of the action of operator $\tilde{H}_{1}$ ) The Fourier transform of the operator $\bar{H}_{1}$ is a bounded self-adjoint operator $\tilde{H}_{1}$ acting in the space $\tilde{\mathcal{H}}_{1}$ be the formula

$$
\begin{aligned}
\left(\tilde{H}_{1} f\right)(\lambda)= & {\left[A+2 B \sum_{i=1}^{v} \cos \lambda_{i}\right] f(\lambda)+\varepsilon_{1} \int_{T^{v}} f(s) \mathrm{d} s } \\
& +2 \varepsilon_{2} \int_{T^{v}} \sum_{i=1}^{v}\left[\cos \lambda_{i}+\cos s_{i}\right] \mathrm{d} s .
\end{aligned}
$$

Comparing the formulas (5) and (8), and using tensor products of Hilbert spaces and tensor products of operators in Hilbert spaces [17], and taking into account that the function $f(\lambda, \mu, \gamma, \theta)$ is an antisymmetric function, we can verify that the operator ${ }^{1} \tilde{H}_{1}^{t}$ can be represented in the form

$$
{ }^{1} \tilde{H}_{1}^{t}=\left\{\tilde{H}_{1} \otimes I+\otimes I \otimes \tilde{H}_{1}+K\right\} \otimes I \otimes I+I \otimes I \otimes\left\{\tilde{H}_{1} \otimes I+I \otimes \tilde{H}_{1}\right\},
$$

where $I$ is the unit operator in space $\tilde{\mathcal{H}}_{1}$, and $K=K_{1 \Lambda_{1}}+K_{2}$, and $\left(K_{1 \Lambda_{1}} f\right)(\lambda)=U \int_{T^{v}} f_{\Lambda_{1}}(s) \mathrm{d} s$, and $\left(K_{2} f\right)(s, t)=\int_{T^{v}} \int_{T^{v}} f(s, t) \mathrm{d} s \mathrm{~d} t$, and $f_{\Lambda_{1}}(s)=f\left(s, \Lambda_{1}-s\right), \Lambda_{1}=\lambda+\theta$. 
It is known that the continuous spectrum of operator $\tilde{H}_{1}$ fills the entire segment $\left[m_{v}, M_{v}\right]=[A-2 B v, A+2 B v]$. We set

$D_{v}(z)=\left(b_{2}-b_{3}\right)^{v-1}\left\{a_{1}\left[b_{2}+(v-1) b_{3}\right]-v a_{2} b_{1}\right\}$, where

$$
\begin{gathered}
a_{1}=1+\int_{T^{v}} \frac{\left[\varepsilon_{1}+2 \varepsilon_{2} \sum_{i=1}^{v} \cos s_{i}\right] \mathrm{d} s_{1} \cdots \mathrm{d} s_{v}}{A+2 B \sum_{i=1}^{v} \cos s_{i}-z}, \\
a_{2}=\int_{T^{v}} \frac{\cos s_{i}\left[\varepsilon_{1}+2 \varepsilon_{2} \sum_{i=1}^{v} \cos s_{i}\right] \mathrm{d} s_{1} \cdots \mathrm{d} s_{v}}{A+2 B \sum_{i=1}^{v} \cos s_{i}-z}, \\
b_{1}=2 \varepsilon_{2} \int_{T^{v}} \frac{\mathrm{d} s_{1} \cdots \mathrm{d} s_{v}}{A+2 B \sum_{i=1}^{v} \cos s_{i}-z}, b_{2}=1+2 \varepsilon_{2} \int_{T^{v}} \frac{\cos s_{i} \mathrm{ds}_{1} \cdots \mathrm{d} s_{v}}{A+2 B \sum_{i=1}^{v} \cos s_{i}-z}, \\
b_{3}=2 \varepsilon_{2} \int_{T^{v}} \frac{\cos s_{i} \mathrm{~d} s_{1} \cdots \mathrm{d} s_{v}}{A+2 B \sum_{i=1}^{v} \cos s_{i}-z},
\end{gathered}
$$

and $v$ is lattice dimensionality.

Lemma 3. A number $z_{0} \notin\left[m_{v}, M_{v}\right]$ is an eigenvalue of operator $\tilde{H}_{1}$ if and only if it is a zero of the function $D_{v}(z)$.

Definition 1. The eigenfunction $\varphi \in L_{2}\left(T^{v}\right)$ of the operator $\tilde{H}_{1}$ corresponding to an eigenvalue $\mathrm{z} \notin\left[m_{v}, M_{v}\right]$ is called a local impurity state (LIS) of $\tilde{H}_{1}$, and $z$ is called the energy of this state.

The following Theorem describes the exchange of the spectrum of operator $\tilde{H}_{1}$ in the case $v=1$.

Theorem 5. (description of the spectra of the operator $\tilde{H}_{1}$ )

A) If $\varepsilon_{2}=-B$ and $\varepsilon_{1}<-2 B$ (respectively, $\varepsilon_{2}=-B$ and $\varepsilon_{1}>2 B$ ), then the operator $\tilde{H}_{1}$ has a unique eigenvalue $z=A+\varepsilon_{1}$, lying below (respectively, above) the continuous spectrum of the operator $\tilde{H}_{1}$.

B) If $\varepsilon_{2}=-2 B$ and $\varepsilon_{1}<0$ or $\varepsilon_{2}=0$ and $\varepsilon_{1}<0$ (respectively, $\varepsilon_{2}=-2 B$ and $\varepsilon_{1}>0$ or $\varepsilon_{2}=0$ and $\left.\varepsilon_{1}>0\right)$, then the operator $\tilde{H}_{1}$ has a unique eigenvalue $\mathrm{Z}=A-\sqrt{4 B^{2}+\varepsilon_{1}^{2}}$ (respectively, $\mathrm{Z}=A+\sqrt{4 B^{2}+\varepsilon_{1}^{2}}$ ), lying the below (respectively, above) of continuous spectrum of the operator $\tilde{H}_{1}$.

C) If $\varepsilon_{1}=0$ and $\varepsilon_{2}>0$ (respectively, $\varepsilon_{1}=0$ and $\varepsilon_{2}<-2 B$ ), then the operator $\tilde{H}_{1}$ has a unique eigenvalue $z=A-\frac{2 B E}{\sqrt{E^{2}-1}}$, (respectively, $\left.z=A+\frac{2 B E}{\sqrt{E^{2}-1}}\right)$, where $E=\frac{\left(B+\varepsilon_{2}\right)^{2}}{\varepsilon_{2}^{2}+2 B \varepsilon_{2}}$, lying the below (respectively, above) of continuous spectrum of the operator $\tilde{H}_{1}$.

D) If $\varepsilon_{1}=\frac{2\left(\varepsilon_{2}^{2}+2 B \varepsilon_{2}\right)}{B}$ (respectively, $\varepsilon_{1}=-\frac{2\left(\varepsilon_{2}^{2}+2 B \varepsilon_{2}\right)}{B}$,) then the operator $\tilde{H}_{1}$ has a unique eigenvalue $z=A+\frac{2 B\left(E^{2}+1\right)}{E^{2}-1}$ (respectively, $\left.Z=A-\frac{2 B\left(E^{2}+1\right)}{E^{2}-1}\right)$, where $E=\frac{\left(B+\varepsilon_{2}\right)^{2}}{\varepsilon_{2}^{2}+2 B \varepsilon_{2}}$, lying the above (respectively, below) 
of continuous spectrum of the operator $\tilde{H}_{1}$.

E) If $\varepsilon_{2}>0$ and $\varepsilon_{1}>\frac{2\left(\varepsilon_{2}^{2}+2 B \varepsilon_{2}\right)}{B}$ (respectively, $\varepsilon_{2}<-2 B$ and $\left.\varepsilon_{1}>\frac{2\left(\varepsilon_{2}^{2}+2 B \varepsilon_{2}\right)}{B}\right)$, then the operator $\tilde{H}_{1}$ has a unique eigenvalue $z_{1}=A+\frac{2 B\left(\alpha+E \sqrt{E^{2}-1+\alpha^{2}}\right)}{E^{2}-1}$, where $E=\frac{\left(B+\varepsilon_{2}\right)^{2}}{\varepsilon_{2}^{2}+2 B \varepsilon_{2}}$, and the real number $\alpha>1$, lying the above of continuous spectrum of the operator $\tilde{H}_{1}$.

F) If $\varepsilon_{2}>0$ and $\varepsilon_{1}<-\frac{2\left(\varepsilon_{2}^{2}+2 B \varepsilon_{2}\right)}{B}$ (respectively, $\varepsilon_{2}<-2 B$ and $\left.\varepsilon_{1}<-\frac{2\left(\varepsilon_{2}^{2}+2 B \varepsilon_{2}\right)}{B}\right)$, then the operator $\tilde{H}_{1}$ has a unique eigenvalue $z_{1}=A-\frac{2 B\left(\alpha+E \sqrt{E^{2}-1+\alpha^{2}}\right)}{E^{2}-1}$, where $E=\frac{\left(B+\varepsilon_{2}\right)^{2}}{\varepsilon_{2}^{2}+2 B \varepsilon_{2}}$, and the real number $\alpha>1$, lying the below of continuous spectrum of the operator $\tilde{H}_{1}$.

K) If $\varepsilon_{2}>0$ and $-\frac{2\left(\varepsilon_{2}^{2}+2 B \varepsilon_{2}\right)}{B}<\varepsilon_{1}<\frac{2\left(\varepsilon_{2}^{2}+2 B \varepsilon_{2}\right)}{B}$ (respectively, $\varepsilon_{2}<-2 B$ and $\left.-\frac{2\left(\varepsilon_{2}^{2}+2 B \varepsilon_{2}\right)}{B}<\varepsilon_{1}<\frac{2\left(\varepsilon_{2}^{2}+2 B \varepsilon_{2}\right)}{B}\right)$, then the operator $\tilde{H}_{1}$ has a exactly two eigenvalues $z_{1}=A+\frac{2 B\left(\alpha+E \sqrt{E^{2}-1+\alpha^{2}}\right)}{E^{2}-1}<m_{1}$, and $z_{2}=A+\frac{2 B\left(\alpha-E \sqrt{E^{2}-1+\alpha^{2}}\right)}{E^{2}-1}>M_{1}$, where $E=\frac{\left(B+\varepsilon_{2}\right)^{2}}{\varepsilon_{2}^{2}+2 B \varepsilon_{2}}$, and real number $|\alpha|<1$, lying the above and below of continuous spectrum of the operator $\tilde{H}_{1}$.

M) If $-2 B<\varepsilon_{2}<0$, then the operator $\tilde{H}_{1}$ has no eigenvalue lying the outside of continuous spectrum of the operator $\tilde{H}_{1}$.

Proof. In the case $v=1$, the continuous spectrum of the operator $\tilde{H}_{1}$ coincide with the segment $\left[m_{1}, M_{1}\right]=[A-2 B, A+2 B]$. Expressing all integrals in the equation $D_{1}(z)=0$ through the integral $J(z)=\int_{T} \frac{\mathrm{d} s}{A+2 B \cos s-z}$, we find that the equation $D_{1}(z)=0$ is equivalent to the equation

$$
\left[\varepsilon_{1} B^{2}+\left(\varepsilon_{2}^{2}+2 B \varepsilon_{2}\right)(z-A)\right] J(z)+\left(B+\varepsilon_{2}\right)^{2}=0 .
$$

Moreover, the function $J(z)$ is a differentiable function on the set $\mathbb{R} \backslash\left[m_{1}, M_{1}\right]$, in addition, $J^{\prime}(z)=\int_{T} \frac{\mathrm{d} s}{[A+2 B \cos s-z]^{2}}>0, \quad z \notin\left[m_{1}, M_{1}\right]$. Thus the function $J(z)$ is a monotone increasing function on $\left(-\infty, m_{1}\right)$ and on $\left(M_{1},+\infty\right)$. Furthermore, $J(z) \rightarrow+0$ as $z \rightarrow-\infty, \quad J(z) \rightarrow+\infty$ as $z \rightarrow m_{1}-0, \quad J(z) \rightarrow-\infty$ as $z \rightarrow M_{1}+0$, and $J(z) \rightarrow-0$ as $z \rightarrow+\infty$.

If $\varepsilon_{1} B^{2}+\left(\varepsilon_{2}^{2}+2 B \varepsilon_{2}\right)(z-A) \neq 0$ then from (10) follows that 


$$
J(z)=-\frac{\left(B+\varepsilon_{2}\right)^{2}}{\varepsilon_{1} B^{2}+\left(\varepsilon_{2}^{2}+2 B \varepsilon_{2}\right)(z-A)} .
$$

The function $\psi(z)=-\frac{\left(B+\varepsilon_{2}\right)^{2}}{\varepsilon_{1} B^{2}+\left(\varepsilon_{2}^{2}+2 B \varepsilon_{2}\right)(z-A)}$ has a point of asymptotic discontinuity $z_{0}=A-\frac{B^{2} \varepsilon_{1}}{\varepsilon_{2}^{2}+2 B \varepsilon_{2}}$. Since $\psi^{\prime}(z)=\frac{\left(B+\varepsilon_{2}\right)^{2}\left(\varepsilon_{2}^{2}+2 B \varepsilon_{2}\right)}{\left[\varepsilon_{1} B^{2}+\left(\varepsilon_{2}^{2}+2 B \varepsilon_{2}\right)(z-A)\right]^{2}}$

for all $z \neq z_{0}$ it follows that the function $\psi(z)$ is a monotone increasing (decreasing) function on $\left(-\infty, z_{0}\right)$ and on $\left(z_{0},+\infty\right)$ in the case $\varepsilon_{2}^{2}+2 B \varepsilon_{2}>0$ (respectively, $\varepsilon_{2}^{2}+2 B \varepsilon_{2}<0$ ), in addition, and if $\varepsilon_{2}>0$, or $\varepsilon_{2}<-2 B$, then $\psi(z) \rightarrow+0 \quad$ as $\quad z \rightarrow-\infty, \quad \psi(z) \rightarrow+\infty \quad$ as $\quad z \rightarrow z_{0}-0, \quad \psi(z) \rightarrow-\infty \quad$ as $z \rightarrow z_{0}+0, \quad \psi(z) \rightarrow-0$ as $z \rightarrow+\infty$ (respectively, if $-2 B<\varepsilon_{2}<0$, then $\psi(z) \rightarrow-0 \quad$ as $\quad z \rightarrow-\infty \quad \psi(z) \rightarrow-\infty \quad$ as $\quad z \rightarrow z_{0}-0, \quad \psi(z) \rightarrow+\infty \quad$ as $z \rightarrow z_{0}+0, \psi(z) \rightarrow+0$ as $\left.z \rightarrow+\infty\right)$.

A) If $\varepsilon_{2}=-B$ and $\varepsilon_{1}<-2 B$ (respectively, $\varepsilon_{2}=-B$ and $\varepsilon_{1}>2 B$ ), then the equation for eigenvalues and eigenfunctions (10) has the form

$$
\left\{\varepsilon_{1} B^{2}-B^{2}(z-A)\right\} J(z)=0 .
$$

It is clear, that $J(z) \neq 0$ for the values $z \notin \sigma_{\text {cont }}\left(\tilde{H}_{1}\right)$. Therefore, $\varepsilon_{1}-z+A=0$, i.e., $z=A+\varepsilon_{1}$. If $\varepsilon_{1}<-2 B$, then this eigenvalue lying the below of continuous spectrum of the operator $\tilde{H}_{1}$, if $\varepsilon_{1}>2 B$, then this eigenvalue lying the above of continuous spectrum of the operator $\tilde{H}_{1}$.

B) If $\varepsilon_{2}=-2 B$ and $\varepsilon_{1}<0$ (respectively, $\varepsilon_{2}=-2 B$ and $\varepsilon_{1}>0$ ), then the equation for the eigenvalues and eigenfunctions has the form $\varepsilon_{1} B^{2} J(z)+B^{2}=0$, that is, $J(z)=-\frac{1}{\varepsilon_{1}}$. It is clear, what the integral $J(z)$ calculated in a quadrature, of the below (above) of continuous spectrum of the operator $\tilde{H}_{1}$, the integral $J(z)>0,\left(J(z)<0\right.$,) consequently, $\varepsilon_{1}<0 \quad\left(\varepsilon_{1}>0\right.$.) The calculated the integral $J(z)=\int_{T^{V}} \frac{\mathrm{d} s}{A+2 B \cos s-z}$, the below of continuous spectrum of the operator $\tilde{H}_{1}$, we have the equation of the form $\frac{1}{\sqrt{(A-z)^{2}-4 B^{2}}}=-\frac{1}{\varepsilon_{1}}$. This equation has a solution $z=A-\sqrt{\varepsilon_{1}^{2}+4 B^{2}}$, lying the below continuous spectrum of the operator $\tilde{H}_{1}$. In the above continuous spectrum of the operator $\tilde{H}_{1}$, the equation takes the form $-\frac{1}{\sqrt{(z-A)^{2}-4 B^{2}}}=-\frac{1}{\varepsilon_{1}}$. This equation has a solution of the form $z=A+\sqrt{\varepsilon_{1}^{2}+4 B^{2}}$, lying the above continuous spectrum of the operator $\tilde{H}_{1}$.

C) If $\varepsilon_{1}=0$ and $\varepsilon_{2}>0$ (respectively, $\varepsilon_{1}=0$ and $\varepsilon_{2}<-2 B$ ), then the equation for the eigenvalues and eigenfunctions take in the form

$$
\left(\varepsilon_{2}^{2}+2 B \varepsilon_{2}\right)(z-A) J(z)=-\left(B+\varepsilon_{2}\right)^{2} \text {, or } J(z)=-\frac{\left(B+\varepsilon_{2}\right)^{2}}{\left(\varepsilon_{2}^{2}+2 B \varepsilon_{2}\right)(z-A)} \text {. }
$$


Denote $E=\frac{\left(B+\varepsilon_{2}\right)^{2}}{\varepsilon_{2}^{2}+2 B \varepsilon_{2}}$. Then $J(z)=-\frac{E}{z-A}$, or $J(z)=\frac{E}{A-z}$. In the below of continuous spectrum of the operator $\tilde{H}_{1}$, we have the equation of the form $\frac{1}{\sqrt{(A-z)^{2}-4 B^{2}}}=\frac{E}{A-z}$. This equation has a solution $z=A-\frac{2 B E}{\sqrt{E^{2}-1}}$. It is obviously, that $E^{2}>1$. This eigenvalue lies the below continuous spectrum of the operator $\tilde{H}_{1}$. In the above continuous spectrum of the operator $\tilde{H}_{1}$, the equation for the eigenvalues and eigenfunctions has the form

$-\frac{1}{\sqrt{(z-A)^{2}-4 B^{2}}}=-\frac{E}{z-A}$. From here, we find $z=A+\frac{2 B E}{\sqrt{E^{2}-1}}$. This eigenvalue lies the above continuous spectrum of the operator $\tilde{H}_{1}$.

D) If $\varepsilon_{1}=\frac{2\left(\varepsilon_{2}^{2}+2 B \varepsilon_{2}\right)}{B}$, then the equation for eigenvalues and eigenfunctions has the form $\left(\varepsilon_{2}^{2}+2 B \varepsilon_{2}\right)(z-A+2 B) J(z)=-\left(B+\varepsilon_{2}\right)^{2}$, from this

$$
J(z)=-\frac{\left(B+\varepsilon_{2}\right)^{2}}{\left(\varepsilon_{2}^{2}+2 B \varepsilon_{2}\right)(z-A+2 B)} .
$$

We denote $E=\frac{\left(B+\varepsilon_{2}\right)^{2}}{\varepsilon_{2}^{2}+2 B \varepsilon_{2}}$. In the first, we consider Equation (12) in the below continuous spectrum of the operator $\tilde{H}_{1}$. In Equation (12) we find the equation of the form $\frac{1}{\sqrt{(A-z)^{2}-4 B^{2}}}=\frac{E}{A-z-2 B}$. From this, we find $z_{1}=A+\frac{2 B\left(E^{2}+1\right)}{E^{2}-1}$, and $z_{2}=A-2 B$. Now we verify the conditions $z_{i}<A-2 B, i=1,2$. The inequality $z_{1}<A-2 B$, is incorrect, and inequality $z_{2}<A-2 B$, is incorrect. We now consider Equation (12) in the above continuous spectrum of the operator $\tilde{H}_{1}$. We have $-\frac{1}{\sqrt{(z-A)^{2}-4 B^{2}}}=-\frac{E}{z-A+2 B}$. From this equation, we find the same solutions $z_{1}$ and $z_{2}$, the outside of the domain of continuous spectrum of the operator $\tilde{H}_{1}$. Now we verify the conditions $z_{i}>A+2 B, i=1,2$. The inequality $z_{1}>A+2 B$, is correct, and inequality $z_{2}>A+2 B$, is incorrect. Consequently, in this case, the operator $\tilde{H}_{1}$ has a unique eigenvalue $z_{1}=A+\frac{2 B\left(E^{2}+1\right)}{E^{2}-1}$, lying the above continuous spectrum of the operator $\tilde{H}_{1}$.

Let $\varepsilon_{1}=-\frac{2\left(\varepsilon_{2}^{2}+2 B \varepsilon_{2}\right)}{B}$, then the equation of eigenvalues and eigenfunctions take in the form $J(z)=-\frac{E}{z-A-2 B}$, where $E=\frac{\left(B+\varepsilon_{2}\right)^{2}}{\varepsilon_{2}^{2}+2 B \varepsilon_{2}}$.

In the below of continuous spectrum of the operator $\tilde{H}_{1}$, we have equation of 
the form $\frac{1}{\sqrt{(A-z)^{2}-4 B^{2}}}=\frac{E}{A-z+2 B}$. From here we find $z_{1}=A-\frac{2 B\left(E^{2}+1\right)}{E^{2}-1}$, and $z_{2}=A+2 B$. Inequalities $z_{1}<A-2 B$, is correct, and $z_{2}<A-2 B$, is incorrect. In the above continuous spectrum of the operator $\tilde{H}_{1}$, we have equation of the form $-\frac{1}{\sqrt{(z-A)^{2}-4 B^{2}}}=-\frac{E}{z-A-2 B}$. It follows that what $z_{1}=A-\frac{2 B\left(E^{2}+1\right)}{E^{2}-1}$, and $z_{2}=A+2 B$. The inequality $z_{1}>A+2 B$, and $z_{2}>A+2 B$, are incorrect. Therefore, in this case, the operator $\tilde{H}_{1}$ has a unique eigenvalue $z_{1}=A-\frac{2 B\left(E^{2}+1\right)}{E^{2}-1}$, lying the below continuous spectrum of the operator $\tilde{H}_{1}$.

E) If $\varepsilon_{2}>0$ and $\varepsilon_{1}>\frac{2\left(\varepsilon_{2}^{2}+2 B \varepsilon_{2}\right)}{B}$, (respectively, $\varepsilon_{2}<-2 B$ and $\left.\varepsilon_{1}>\frac{2\left(\varepsilon_{2}^{2}+2 B \varepsilon_{2}\right)}{B}\right)$, then consider necessary, that $\varepsilon_{1}=\alpha \times \frac{2\left(\varepsilon_{2}^{2}+2 B \varepsilon_{2}\right)}{B}$, where $\alpha>1$-real number. Then the equation for eigenvalues and eigenfunctions has the form

$$
\begin{gathered}
\left\{\alpha \times \frac{2\left(\varepsilon_{2}^{2}+2 B \varepsilon_{2}\right)}{B} \times B^{2}+\left(\varepsilon_{2}^{2}+2 B \varepsilon_{2}\right)(z-A)\right\} J(z)+\left(B+\varepsilon_{2}\right)^{2}=0, \\
\text { or }\left(\varepsilon_{2}^{2}+2 B \varepsilon_{2}\right)(z-A+2 \alpha B) J(z)+\left(B+\varepsilon_{2}\right)^{2}=0 \text {. From this } \\
J(z)=-\frac{\left(B+\varepsilon_{2}\right)^{2}}{\left(\varepsilon_{2}^{2}+2 B \varepsilon_{2}\right)(z-A+2 \alpha B)} .
\end{gathered}
$$

We denote $E=\frac{\left(B+\varepsilon_{2}\right)^{2}}{\varepsilon_{2}^{2}+2 B \varepsilon_{2}}$, then $J(z)=-\frac{E}{z-A+2 \alpha B}$. In the first, we consider this equation in the below continuous spectrum of the operator $\tilde{H}_{1}$. Then $\frac{1}{\sqrt{(A-z)^{2}-4 B^{2}}}=\frac{E}{A-z-2 \alpha B}$. This equation has the solutions
$z_{1}=A+\frac{2 B\left(\alpha+E \sqrt{E^{2}-1+\alpha^{2}}\right)}{E^{2}-1}$, and $z_{2}=A+\frac{2 B\left(\alpha-E \sqrt{E^{2}-1+\alpha^{2}}\right)}{E^{2}-1}$.

Now, we verify the condition $z_{i}<A-2 B, i=1,2$. The solution $z_{1}$ no satisfies the condition $z_{1}<A-2 B$, but $z_{2}$ satisfies the condition $z_{2}<A-2 B$. We now verify the conditions $z_{2}<A-2 \alpha B$. The appear, this inequality is incorrect. The appear inequalities $z_{1}>A+2 B$ is correct, and $z_{2}>A+2 B$, is incorrect. We now verify the conditions $z_{1}>A-2 \alpha B$. So far as, $A-2 \alpha B<A+2 B$, the appear, this inequality is correct. Consequently, in this case, the operator $\tilde{H}_{1}$ has a unique eigenvalue $z_{1}=A+\frac{2 B\left(\alpha+E \sqrt{E^{2}-1+\alpha^{2}}\right)}{E^{2}-1}$, above of continuous 
spectrum of the operator $\tilde{H}_{1}$.

F) If $\varepsilon_{2}>0$, and $\varepsilon_{1}<-\frac{2\left(\varepsilon_{2}^{2}+2 B \varepsilon_{2}\right)}{B}$, (respectively, $\varepsilon_{2}<-2 B$, and $\left.\varepsilon_{1}<-\frac{2\left(\varepsilon_{2}^{2}+2 B \varepsilon_{2}\right)}{B}\right)$, then we assume that $\varepsilon_{1}=-\alpha \times \frac{2\left(\varepsilon_{2}^{2}+2 B \varepsilon_{2}\right)}{B}$, where $\alpha>1$-real number. The equation for eigenvalues and eigenfunctions take in the form

$$
\left(\varepsilon_{2}^{2}+2 B \varepsilon_{2}\right)(z-A-2 \alpha B) J(z)=-\left(B+\varepsilon_{2}\right)^{2} .
$$

From here $J(z)=-\frac{\left(B+\varepsilon_{2}\right)^{2}}{\left(\varepsilon_{2}^{2}+2 B \varepsilon_{2}\right)(z-A-2 \alpha B)}$. The introduce notation $E=\frac{\left(B+\varepsilon_{2}\right)^{2}}{\varepsilon_{2}^{2}+2 B \varepsilon_{2}}$. Then

$$
J(z)=-\frac{E}{z-A-2 \alpha B} .
$$

In the below continuous spectrum of the operator $\tilde{H}_{1}$, we have the equation

$$
J(z)=\frac{E}{A-z+2 \alpha B}, \text { from here } \frac{1}{\sqrt{(A-z)^{2}-4 B^{2}}}=\frac{E}{A-z+2 \alpha B} ;
$$

This equation takes the form

$$
\left(E^{2}-1\right)(A-z)^{2}-4 \alpha B(A-z)-4 B^{2}\left(E^{2}+\alpha^{2}\right)=0 .
$$

We find

$$
z_{1}=A-\frac{2 B\left(\alpha+E \sqrt{E^{2}-1+\alpha^{2}}\right)}{E^{2}-1} \text { and } z_{2}=A-\frac{2 B\left(\alpha-E \sqrt{E^{2}-1+\alpha^{2}}\right)}{E^{2}-1} .
$$

We now verify the conditions $z_{i}<m_{1}=A-2 B, i=1,2$. The appear, that $z_{1}<A-2 B$, is correctly and $z_{2}<A-2 B$, is incorrect. Now we consider Equation (13) in the above continuous spectrum of the operator $\tilde{H}_{1}$. Then

$$
\begin{gathered}
J(z)=-\frac{E}{z-A-2 \alpha B} . \text { From this }-\frac{1}{\sqrt{(z-A)^{2}-4 B^{2}}}=-\frac{E}{z-A-2 \alpha B} . \text { We find } \\
z_{1}=A-\frac{2 B\left(\alpha+E \sqrt{E^{2}-1+\alpha^{2}}\right)}{E^{2}-1}, \text { and } z_{2}=A+\frac{2 B\left(-\alpha+E \sqrt{E^{2}-1+\alpha^{2}}\right)}{E^{2}-1} .
\end{gathered}
$$

We verify the conditions $z_{i}>A+2 B, i=1,2$. The appear $z_{1}>A+2 B$, it is not true, and the $z_{2}>A+2 B$, is true. We now verify the conditions $z_{2}>A+2 \alpha B$. The appear, this inequality is incorrect. Consequently, in this case, the operator $\tilde{H}_{1}$ have unique eigenvalue $z_{1}=A-\frac{2 B\left(\alpha+E \sqrt{E^{2}-1+\alpha^{2}}\right)}{E^{2}-1}<m_{1}$, i.e., lying the below continuous spectrum of the operator $\tilde{H}_{1}$.

K) If $\varepsilon_{2}>0$ and $-\frac{2\left(\varepsilon_{2}^{2}+2 B \varepsilon_{2}\right)}{B}<\varepsilon_{1}<\frac{2\left(\varepsilon_{2}^{2}+2 B \varepsilon_{2}\right)}{B}$ (respectively, 
$\varepsilon_{2}<-2 B$ and $\left.-\frac{2\left(\varepsilon_{2}^{2}+2 B \varepsilon_{2}\right)}{B}<\varepsilon_{1}<\frac{2\left(\varepsilon_{2}^{2}+2 B \varepsilon_{2}\right)}{B}\right)$, we take

$\varepsilon_{1}=\alpha \times \frac{2\left(\varepsilon_{2}^{2}+2 B \varepsilon_{2}\right)}{B}$, where $-1<\alpha<1$-real number. Then the equation for eigenvalues and eigenfunctions has the form

$$
\left(\varepsilon_{2}^{2}+2 B \varepsilon_{2}\right)(z-A+2 \alpha B) J(z)=-\left(B+\varepsilon_{2}\right)^{2},|\alpha|<1 .
$$

We denote $E=\frac{\left(B+\varepsilon_{2}\right)^{2}}{\varepsilon_{2}^{2}+2 B \varepsilon_{2}}$. Then Equation (14) receives the form

$$
J(z)=-\frac{E}{z-A+2 \alpha B} .
$$

In the below of continuous spectrum of the operator $\tilde{H}_{1}$ we have the equation of the form

$$
\frac{1}{\sqrt{(A-z)^{2}-4 B^{2}}}=\frac{E}{A-z-2 \alpha B},|\alpha|<1
$$

This equation has a solutions

$$
z_{1}=A+\frac{2 B\left(\alpha+E \sqrt{E^{2}-1+\alpha^{2}}\right)}{E^{2}-1} \text {, and } z_{2}=A+\frac{2 B\left(\alpha-E \sqrt{E^{2}-1+\alpha^{2}}\right)}{E^{2}-1} \text {. }
$$

The inequalities $z_{1}<A-2 B$, and $z_{1}<A-2 \alpha B$, is incorrect. The inequalities $z_{2}<A-2 B$, is correct. We now verify the conditions $z_{2}<A-2 \alpha B$, since $A-2 B<A-2 \alpha B$, this inequality is true. We now consider Equation (14) in the above continuous spectrum of the operator $\tilde{H}_{1}$. We have the equation of the form

$$
-\frac{1}{\sqrt{(z-A)^{2}-4 B^{2}}}=-\frac{E}{z-A+2 \alpha B} .
$$

This equation has a solutions

$$
z_{1}=A+\frac{2 B\left(\alpha+E \sqrt{E^{2}-1+\alpha^{2}}\right)}{E^{2}-1} \text {, and } z_{2}=A+\frac{2 B\left(\alpha-E \sqrt{E^{2}-1+\alpha^{2}}\right)}{E^{2}-1} \text {. }
$$

The inequalities $z_{1}>A+2 B$, and $z_{1}>A-2 \alpha B$ is true, as $A+2 B>A-2 \alpha B$, that the inequality $z_{1}>A-2 \alpha B$ is correct. The inequalities $z_{2}>A+2 B$, and $z_{2}>A+2 \alpha B$ is incorrect. Consequently, in this case, the operator $\tilde{H}_{1}$ has exactly two eigenvalues

$$
z_{1}=A+\frac{2 B\left(\alpha+E \sqrt{E^{2}-1+\alpha^{2}}\right)}{E^{2}-1} \text {, and } z_{2}=A+\frac{2 B\left(\alpha-E \sqrt{E^{2}-1+\alpha^{2}}\right)}{E^{2}-1} \text {, }
$$

lying the above and below of continuous spectrum of the operator $\tilde{H}_{1}$.

M) If $-2 B<\varepsilon_{2}<0$, then $\varepsilon_{2}^{2}+2 B \varepsilon_{2}<0$, and the function $\psi(z)=-\frac{\left(B+\varepsilon_{2}\right)^{2}}{\varepsilon_{1} B+\left(\varepsilon_{2}^{2}+2 B \varepsilon_{2}\right)(z-A)}$ is a decreasing function in the intervals 
$\left(-\infty, z_{0}\right)$ and $\left(z_{0},+\infty\right)$; By, $z \rightarrow-\infty$ the function $\psi(z) \rightarrow-0$, and by $z \rightarrow z_{0}-0$, the function $\psi(z) \rightarrow-\infty$, and by $z \rightarrow+\infty, \psi(z) \rightarrow+0$, and by $z \rightarrow z_{0}+0, \psi(z) \rightarrow+\infty$. The function $J(z) \rightarrow 0$, by $z \rightarrow-\infty$, and by $z \rightarrow m_{1}-0$, the function $J(z) \rightarrow+\infty$, and by $z \rightarrow M_{1}+0$, the function $J(z) \rightarrow-\infty$, by $z \rightarrow+\infty$, the function $J(z) \rightarrow-0$. Therefore, the equation $\psi(z)=J(z)$, that's impossible the solutions in the outside the continuous spectrum of operator $\tilde{H}_{1}$. Therefore, in this case, the operator $\tilde{H}_{1}$ has no eigenvalues lying the outside of continuous spectrum of the operator $\tilde{H}_{1}$.

Consequently, the spectrum of operator $\tilde{H}_{1}$ is consisted of a continuous spectrum and at most two eigenvalues.

Now, using the obtained results and representation (9), we describe the structure of essential spectrum and discrete spectrum of the energy operator of four-electron systems in the impurity Hubbard model in the first triplet state.

Theorem 6. (essential spectra of the operator ${ }^{1} \tilde{H}_{1}^{t}$ ) Let $v=1$. Then

A) If $\varepsilon_{2}=-B$ and $\varepsilon_{1}<-2 B$ (respectively, $\varepsilon_{2}=-B$ and $\varepsilon_{1}>2 B$ ), then the essential spectrum of the operator ${ }^{1} \tilde{H}_{1}^{t}$ is consists of the union of $N_{1}$ segments, where $4 \leq N_{1} \leq 8$ :

$$
\begin{aligned}
\sigma_{\text {ess }}\left({ }^{1} \tilde{H}_{1}^{t}\right)= & {[4 A-8 B, 4 A+8 B] \cup[3 A-6 B+z, 3 A+6 B+z] } \\
& \cup[2 A-4 B+2 z, 2 A+4 B+2 z] \cup[A-2 B+3 z, A+2 B+3 z] \\
& \cup\left[2 A-4 B+z_{3}, 2 A+4 B+z_{3}\right] \cup\left[2 A-4 B+z_{4}, 2 A+4 B+z_{4}\right] \\
& \cup\left[A-2 B+z+z_{3}, A+2 B+z+z_{3}\right] \\
& \cup\left[A-2 B+z+z_{4}, A+2 B+z+z_{4}\right],
\end{aligned}
$$

and the discrete spectrum of the operator ${ }^{1} \tilde{H}_{1}^{t}$ is consists of no more than three eigenvalues:

$$
\sigma_{\text {disc }}\left({ }^{1} \tilde{H}_{1}^{t}\right)=\left\{4 z, 2 z+z_{3}, 2 z+z_{4}\right\},
$$

where $z=A+\varepsilon_{1}$, and $Z_{3}$ and $z_{4}$ are the additional eigenvalues of operator ${ }^{1} \tilde{H}_{1}^{t}$.

B) If $\varepsilon_{2}=-2 B$ and $\varepsilon_{1}<0$ or $\varepsilon_{2}=0$ and $\varepsilon_{1}<0$ (respectively, $\varepsilon_{2}=-B$ and $\varepsilon_{1}>0$ or $\varepsilon_{2}=0$ and $\varepsilon_{1}>0$ ), then the essential spectrum of the operator ${ }^{1} \tilde{H}_{1}^{t}$ is consists of the union of $N_{1}$ segments, where $4 \leq N_{1} \leq 8$ :

$$
\begin{aligned}
\sigma_{\text {ess }}\left({ }^{1} \tilde{H}_{1}^{t}\right)= & {[4 A-8 B, 4 A+8 B] \cup[3 A-6 B+z, 3 A+6 B+z] } \\
& \cup[2 A-4 B+2 z, 2 A+4 B+2 z] \cup[A-2 B+3 z, A+2 B+3 z] \\
& \cup\left[2 A-4 B+z_{3}, 2 A+4 B+z_{3}\right] \cup\left[2 A-4 B+z_{4}, 2 A+4 B+z_{4}\right] \\
& \cup\left[A-2 B+z+z_{3}, A+2 B+z+z_{3}\right] \\
& \cup\left[A-2 B+z+z_{4}, A+2 B+z+z_{4}\right],
\end{aligned}
$$

and discrete spectrum of the operator ${ }^{1} \tilde{H}_{1}^{t}$ is consists of no more three eigenvalues:

$$
\sigma_{\text {disc }}\left({ }^{1} \tilde{H}_{1}^{t}\right)=\left\{4 z, 2 z+z_{3}, 2 z+z_{4}\right\},
$$

where $z=A+\sqrt{4 B^{2}+\varepsilon_{1}^{2}}$, and $z_{3}$ and $z_{4}$ are the additional eigenvalues of 
operator ${ }^{1} \tilde{H}_{1}^{t}$.

C) If $\varepsilon_{1}=0$ and $\varepsilon_{2}>0$ (respectively, $\varepsilon_{1}=0$ and $\varepsilon_{2}<-2 B$ ), then the essential spectrum of the operator ${ }^{1} \tilde{H}_{1}^{t}$ is consists of the union of $N_{1}$ segments, where $4 \leq N_{1} \leq 8$ :

$$
\begin{aligned}
\sigma_{\text {ess }}\left({ }^{1} \tilde{H}_{1}^{t}\right)= & {[4 A-8 B, 4 A+8 B] \cup[3 A-6 B+z, 3 A+6 B+z] } \\
& \cup[2 A-4 B+2 z, 2 A+4 B+2 z] \cup[A-2 B+3 z, A+2 B+3 z] \\
& \cup\left[2 A-4 B+z_{3}, 2 A+4 B+z_{3}\right] \cup\left[2 A-4 B+z_{4}, 2 A+4 B+z_{4}\right] \\
& \cup\left[A-2 B+z+z_{3}, A+2 B+z+z_{3}\right] \\
& \cup\left[A-2 B+z+z_{4}, A+2 B+z+z_{4}\right],
\end{aligned}
$$

and discrete spectrum of the operator ${ }^{1} \tilde{H}_{1}^{t}$ is consists of no more than three eigenvalues.

$$
\sigma_{\text {disc }}\left({ }^{1} \tilde{H}_{1}^{t}\right)=\left\{4 z, 2 z+z_{3}, 2 z+z_{4}\right\},
$$

where $z=A-\frac{2 B E}{\sqrt{E^{2}-1}},\left(\right.$ respectively, $\left.z=A+\frac{2 B E}{\sqrt{E^{2}-1}}\right)$, and $E=\frac{\left(B+\varepsilon_{2}\right)^{2}}{\varepsilon_{2}^{2}+2 B \varepsilon_{2}}$, and $z_{3}$ and $z_{4}$ are the additional eigenvalues of operator ${ }^{1} \tilde{H}_{1}^{t}$.

D) If $\varepsilon_{1}=\frac{2\left(\varepsilon_{2}^{2}+2 B \varepsilon_{2}\right)}{B}$ (respectively, $\varepsilon_{1}=-\frac{2\left(\varepsilon_{2}^{2}+2 B \varepsilon_{2}\right)}{B}$,) then the essential spectrum of the operator ${ }^{1} \tilde{H}_{1}^{t}$ is consists of the union of $N_{1}$ segments, where $4 \leq N_{1} \leq 8$ :

$$
\begin{aligned}
\sigma_{\text {ess }}\left({ }^{1} \tilde{H}_{1}^{t}\right)= & {[4 A-8 B, 4 A+8 B] \cup[3 A-6 B+z, 3 A+6 B+z] } \\
& \cup[2 A-4 B+2 z, 2 A+4 B+2 z] \cup[A-2 B+3 z, A+2 B+3 z] \\
& \cup\left[2 A-4 B+z_{3}, 2 A+4 B+z_{3}\right] \cup\left[2 A-4 B+z_{4}, 2 A+4 B+z_{4}\right] \\
& \cup\left[A-2 B+z+z_{3}, A+2 B+z+z_{3}\right] \\
& \cup\left[A-2 B+z+z_{4}, A+2 B+z+z_{4}\right],
\end{aligned}
$$

and discrete spectrum of the operator ${ }^{1} \tilde{H}_{1}^{t}$ is consists of no more than three eigenvalues.

$$
\sigma_{\text {disc }}\left({ }^{1} \tilde{H}_{1}^{t}\right)=\left\{4 z, 2 z+z_{3}, 2 z+z_{4}\right\},
$$

where $z=A+\frac{2 B\left(E^{2}+1\right)}{E^{2}-1}$ (respectively, $\left.z=A-\frac{2 B\left(E^{2}+1\right)}{E^{2}-1}\right)$, and $E=\frac{\left(B+\varepsilon_{2}\right)^{2}}{\varepsilon_{2}^{2}+2 B \varepsilon_{2}}$, and $z_{3}$ and $z_{4}$ are the additional eigenvalues of operator ${ }^{1} \tilde{H}_{1}^{t}$.

E) If $\varepsilon_{2}>0$ and $\varepsilon_{1}>\frac{2\left(\varepsilon_{2}^{2}+2 B \varepsilon_{2}\right)}{B}$ (respectively, $\varepsilon_{2}<-2 B$ and $\left.\varepsilon_{1}>\frac{2\left(\varepsilon_{2}^{2}+2 B \varepsilon_{2}\right)}{B}\right)$, then the essential spectrum of the operator ${ }^{1} \tilde{H}_{1}^{t}$ is consists of the union of $N_{1}$ segments, where $4 \leq N_{1} \leq 8$ :

$$
\begin{aligned}
\sigma_{\text {ess }}\left({ }^{1} \tilde{H}_{1}^{t}\right)= & {[4 A-8 B, 4 A+8 B] \cup[3 A-6 B+z, 3 A+6 B+z] } \\
& \cup[2 A-4 B+2 z, 2 A+4 B+2 z] \cup[A-2 B+3 z, A+2 B+3 z]
\end{aligned}
$$




$$
\begin{aligned}
& \cup\left[2 A-4 B+z_{3}, 2 A+4 B+z_{3}\right] \cup\left[2 A-4 B+z_{4}, 2 A+4 B+z_{4}\right] \\
& \cup\left[A-2 B+z+z_{3}, A+2 B+z+z_{3}\right] \\
& \cup\left[A-2 B+z+z_{4}, A+2 B+z+z_{4}\right],
\end{aligned}
$$

and discrete spectrum of the operator ${ }^{1} \tilde{H}_{1}^{t}$ is consists of no more than three eigenvalues.

$$
\sigma_{\text {disc }}\left({ }^{1} \tilde{H}_{1}^{t}\right)=\left\{4 z, 2 z+z_{3}, 2 z+z_{4}\right\},
$$

where

$$
z_{1}=A+\frac{2 B\left(\alpha+E \sqrt{E^{2}-1+\alpha^{2}}\right)}{E^{2}-1},
$$

and $E=\frac{\left(B+\varepsilon_{2}\right)^{2}}{\varepsilon_{2}^{2}+2 B \varepsilon_{2}}$, and the real number $\alpha>1$, and $z_{3}$ and $z_{4}$ are the additional eigenvalues of operator ${ }^{1} \tilde{H}_{1}^{t}$.

F) If $\varepsilon_{2}>0$ and $\varepsilon_{1}<-\frac{2\left(\varepsilon_{2}^{2}+2 B \varepsilon_{2}\right)}{B}$ (respectively, $\varepsilon_{2}<-2 B$ and $\left.\varepsilon_{1}<-\frac{2\left(\varepsilon_{2}^{2}+2 B \varepsilon_{2}\right)}{B}\right)$, then the essential spectrum of the operator ${ }^{1} \tilde{H}_{1}^{t}$ is consists of the union of $N_{1}$ segments, where $4 \leq N_{1} \leq 8$ :

$$
\begin{aligned}
\sigma_{\text {ess }}\left(\tilde{H}_{1}^{t}\right)= & {[4 A-8 B, 4 A+8 B] \cup[3 A-6 B+z, 3 A+6 B+z] } \\
& \cup[2 A-4 B+2 z, 2 A+4 B+2 z] \cup[A-2 B+3 z, A+2 B+3 z] \\
& \cup\left[2 A-4 B+z_{3}, 2 A+4 B+z_{3}\right] \cup\left[2 A-4 B+z_{4}, 2 A+4 B+z_{4}\right] \\
& \cup\left[A-2 B+z+z_{3}, A+2 B+z+z_{3}\right] \\
& \cup\left[A-2 B+z+z_{4}, A+2 B+z+z_{4}\right],
\end{aligned}
$$

and discrete spectrum of the operator ${ }^{1} \tilde{H}_{1}^{t}$ is consists of no more than three eigenvalues:

$$
\sigma_{\text {disc }}\left({ }^{1} \tilde{H}_{1}^{t}\right)=\left\{4 z, 2 z+z_{3}, 2 z+z_{4}\right\},
$$

where

$$
z_{1}=A-\frac{2 B\left(\alpha+E \sqrt{E^{2}-1+\alpha^{2}}\right)}{E^{2}-1},
$$

and $E=\frac{\left(B+\varepsilon_{2}\right)^{2}}{\varepsilon_{2}^{2}+2 B \varepsilon_{2}}$, and the real number $\alpha>1$, and $z_{3}$ and $z_{4}$ are the additional eigenvalues of operator ${ }^{1} \tilde{H}_{1}^{t}$.

K) If $\varepsilon_{2}>0$ and $-\frac{2\left(\varepsilon_{2}^{2}+2 B \varepsilon_{2}\right)}{B}<\varepsilon_{1}<\frac{2\left(\varepsilon_{2}^{2}+2 B \varepsilon_{2}\right)}{B}$ (respectively, $\varepsilon_{2}<-2 B$ and $\left.-\frac{2\left(\varepsilon_{2}^{2}+2 B \varepsilon_{2}\right)}{B}<\varepsilon_{1}<\frac{2\left(\varepsilon_{2}^{2}+2 B \varepsilon_{2}\right)}{B}\right)$, then the essential spectrum of the operator ${ }^{1} \tilde{H}_{1}^{t}$ is consists of the union of $N_{1}$ segments, where $10 \leq N_{1} \leq 16$ : 


$$
\begin{aligned}
& \sigma_{\text {ess }}\left({ }^{1} \tilde{H}_{1}^{t}\right)=[4 A-8 B, 4 A+8 B] \cup\left[3 A-6 B+z_{1}, 3 A+6 B+z_{1}\right] \\
& \cup\left[3 A-6 B+z_{2}, 3 A+6 B+z_{2}\right] \cup\left[2 A-4 B+2 z_{1}, 2 A+4 B+2 z_{1}\right] \\
& \cup\left[2 A-4 B+z_{1}+z_{2}, 2 A+4 B+z_{1}+z_{2}\right] \cup\left[2 A-4 B+2 z_{2}, 2 A+4 B+2 z_{2}\right] \\
& \cup\left[A-2 B+3 z_{1}, A+2 B+3 z_{1}\right] \cup\left[A-2 B+2 z_{1}+z_{2}, A+2 B+2 z_{1}+z_{2}\right] \\
& \cup\left[A-2 B+z_{1}+2 z_{2}, A+2 B+z_{1}+2 z_{2}\right] \cup\left[A-2 B+3 z_{2}, A+2 B+3 z_{2}\right] \\
& \cup\left[2 A-4 B+z_{3}, 2 A+4 B+z_{3}\right] \cup\left[2 A-4 B+z_{4}, 2 A+4 B+z_{4}\right] \\
& \cup\left[A-2 B+z_{1}+z_{3}, A+2 B+z_{1}+z_{3}\right] \cup\left[A-2 B+z_{1}+z_{4}, A+2 B+z_{1}+z_{4}\right] \\
& \cup\left[A-2 B+z_{2}+z_{3}, A+2 B+z_{2}+z_{3}\right] \cup\left[A-2 B+z_{2}+z_{4}, A+2 B+z_{2}+z_{4}\right],
\end{aligned}
$$

and discrete spectrum of the operator ${ }^{1} \tilde{H}_{1}^{t}$ is consists of no more than eleven eigenvalues:

$$
\begin{aligned}
\sigma_{\text {disc }}\left({ }^{1} \tilde{H}_{1}^{t}\right)= & \left\{4 z_{1}, 3 z_{1}+z_{2}, 2 z_{1}+2 z_{2}, z_{1}+3 z_{2}, 4 z_{2}, 2 z_{1}+z_{3}, 2 z_{1}+z_{4},\right. \\
& \left.z_{1}+z_{2}+z_{3}, z_{1}+z_{2}+z_{4}, 2 z_{2}+z_{3}, 2 z_{2}+z_{4}\right\},
\end{aligned}
$$

where

$$
z_{1}=A+\frac{2 B\left(\alpha+E \sqrt{E^{2}-1+\alpha^{2}}\right)}{E^{2}-1}<m_{1},
$$

and

$$
z_{2}=A+\frac{2 B\left(\alpha-E \sqrt{E^{2}-1+\alpha^{2}}\right)}{E^{2}-1}>M_{1},
$$

and $E=\frac{\left(B+\varepsilon_{2}\right)^{2}}{\varepsilon_{2}^{2}+2 B \varepsilon_{2}}$, and real number $|\alpha|<1$, and $z_{3}$ and $z_{4}$ are the additional eigenvalues of operator ${ }^{1} \tilde{H}_{1}^{t}$.

M) If $-2 B<\varepsilon_{2}<0$, then the essential spectrum of the operator ${ }^{1} \tilde{H}_{1}^{t}$ is consists of the union of $N_{1}$ segments, where $1 \leq N_{1} \leq 3$ :

$$
\begin{aligned}
\sigma_{\text {ess }}\left({ }^{1} \tilde{H}_{1}^{t}\right)= & {[4 A-8 B, 4 A+8 B] \cup\left[2 A-4 B+z_{3}, 2 A+4 B+z_{3}\right] } \\
& \cup\left[2 A-4 B+z_{4}, 2 A+4 B+z_{4}\right],
\end{aligned}
$$

and the discrete spectrum of operator ${ }^{1} \tilde{H}_{1}^{t}$ is an empty set.

Proof. A) It follows from representation (9), and from Theorem 5, that in one-dimensional case, the continuous spectrum of the operator $\tilde{H}_{1}$ is consisted of $\sigma_{\text {cont }}\left(\tilde{H}_{1}\right)=[A-2 B, A+2 B]$, and discrete spectrum of the operator $\tilde{H}_{1}$ is consists of unique eigenvalue $z$. Therefore, the essential spectrum of the operator ${ }^{1} \tilde{H}_{1}^{t}$ consists of the union of no more than eight segments:

$$
\begin{aligned}
\sigma_{e s s}\left({ }^{1} \tilde{H}_{1}^{t}\right)= & {[4 A-8 B, 4 A+8 B] \cup[3 A-6 B+z, 3 A+6 B+z] } \\
& \cup[2 A-4 B+2 z, 2 A+4 B+2 z] \cup[A-2 B+3 z, A+2 B+3 z] \\
& \cup\left[2 A-4 B+z_{3}, 2 A+4 B+z_{3}\right] \cup\left[2 A-4 B+z_{4}, 2 A+4 B+z_{4}\right] \\
& \cup\left[A-2 B+z+z_{3}, A+2 B+z+z_{3}\right] \\
& \cup\left[A-2 B+z+z_{4}, A+2 B+z+z_{4}\right],
\end{aligned}
$$


and discrete spectrum of the operator ${ }^{1} \tilde{H}_{1}^{t}$ is consists is the no more than three eigenvalues:

$$
\sigma_{\text {disc }}\left({ }^{1} \tilde{H}_{1}^{t}\right)=\left\{4 z, 2 z+z_{3}, 2 z+z_{4}\right\},
$$

This is given to the proof of statement A) from Theorem 6 .

The statements B), C), D), E), F) from Theorem 6 are proved similarly.

We now is proved the statement $\mathrm{K}$ ) from Theorem 6. It can be seen from Theorem 5 (statement $\mathrm{K}$ ) in a one-dimensional case the operator $\tilde{H}_{1}$ has exactly two eigenvalues $z_{1}$ and $z_{2}$ outside the domain of continuous spectrum of the operator $\tilde{H}_{1}$. Therefore, the set $\sigma_{\text {ess }}\left({ }^{1} \tilde{H}_{1}^{t}\right)$ consists of the union of no more than sixteen intervals:

$$
\begin{aligned}
& \sigma_{e s s}\left({ }^{1} \tilde{H}_{1}^{t}\right)=[4 A-8 B, 4 A+8 B] \cup\left[3 A-6 B+z_{1}, 3 A+6 B+z_{1}\right] \\
& \cup\left[3 A-6 B+z_{2}, 3 A+6 B+z_{2}\right] \cup\left[2 A-4 B+2 z_{1}, 2 A+4 B+2 z_{1}\right] \\
& \cup\left[2 A-4 B+z_{1}+z_{2}, 2 A+4 B+z_{1}+z_{2}\right] \cup\left[2 A-4 B+2 z_{2}, 2 A+4 B+2 z_{2}\right] \\
& \cup\left[A-2 B+3 z_{1}, A+2 B+3 z_{1}\right] \cup\left[A-2 B+2 z_{1}+z_{2}, A+2 B+2 z_{1}+z_{2}\right] \\
& \cup\left[A-2 B+z_{1}+2 z_{2}, A+2 B+z_{1}+2 z_{2}\right] \cup\left[A-2 B+3 z_{2}, A+2 B+3 z_{2}\right] \\
& \cup\left[2 A-4 B+z_{3}, 2 A+4 B+z_{3}\right] \cup\left[2 A-4 B+z_{4}, 2 A+4 B+z_{4}\right] \\
& \cup\left[A-2 B+z_{1}+z_{3}, A+2 B+z_{1}+z_{3}\right] \cup\left[A-2 B+z_{1}+z_{4}, A+2 B+z_{1}+z_{4}\right] \\
& \cup\left[A-2 B+z_{2}+z_{3}, A+2 B+z_{2}+z_{3}\right] \cup\left[A-2 B+z_{2}+z_{4}, A+2 B+z_{2}+z_{4}\right] .
\end{aligned}
$$

The discrete spectrum of the operator ${ }^{1} \tilde{H}_{1}^{t}$ is consists of no more than eleven eigenvalues:

$$
\begin{aligned}
\sigma_{\text {disc }}\left({ }^{1} \tilde{H}_{1}^{t}\right)= & \left\{4 z_{1}, 3 z_{1}+z_{2}, 2 z_{1}+2 z_{2}, z_{1}+3 z_{2}, 4 z_{2}, 2 z_{1}+z_{3}, 2 z_{1}+z_{4},\right. \\
& \left.z_{1}+z_{2}+z_{3}, z_{1}+z_{2}+z_{4}, 2 z_{2}+z_{3}, 2 z_{2}+z_{4}\right\},
\end{aligned}
$$

where

$$
z_{1}=A+\frac{2 B\left(\alpha+E \sqrt{E^{2}-1+\alpha^{2}}\right)}{E^{2}-1}<m_{1},
$$

and

$$
z_{2}=A+\frac{2 B\left(\alpha-E \sqrt{E^{2}-1+\alpha^{2}}\right)}{E^{2}-1}>M_{1} \text {, }
$$

and $E=\frac{\left(B+\varepsilon_{2}\right)^{2}}{\varepsilon_{2}^{2}+2 B \varepsilon_{2}}$, and real number $|\alpha|<1$, lying the outside of the essential spectrum of the operator ${ }^{1} \tilde{H}_{1}^{t}, z_{3}$ and $z_{4}$ are the additional eigenvalues of operator ${ }^{1} \tilde{H}_{1}^{t}$. This is given to the proof of statement $\mathrm{K}$ ) from Theorem 6 .

We now are proved the statement $M$ ) from Theorem 6 . It can be seen from Theorem 5 (statement M) in a one-dimensional case the operator $\tilde{H}_{1}$ has no eigenvalues outside of the continuous spectrum of the operator $\tilde{H}_{1}$. Therefore, the set $\sigma_{\text {ess }}\left({ }^{1} \tilde{H}_{1}^{t}\right)$ consists of the union of no more than three segment:

$$
\begin{aligned}
\sigma_{\text {ess }}\left({ }^{1} \tilde{H}_{1}^{t}\right)= & {[4 A-8 B, 4 A+8 B] \cup\left[2 A-4 B+z_{3}, 2 A+4 B+z_{3}\right] } \\
& \cup\left[2 A-4 B+z_{4}, 2 A+4 B+z_{4}\right],
\end{aligned}
$$


and the operator has no eigenvalues, i.e., $\sigma_{\text {disc }}\left({ }^{1} \tilde{H}_{1}^{t}\right)=\varnothing$.

\section{Conflicts of Interest}

The authors declare no conflicts of interest regarding the publication of this paper.

\section{References}

[1] Hubbard, J. (1963) Electron Correlations in Narrow Energy Bands. Proceedings of the Royal Society A, 276, 238-257. https://doi.org/10.1098/rspa.1963.0204

[2] Gutzwiller, M.C. (1963) Effect of Correlation on the Ferromagnetism of Transition Metals. Physical Review Letters, 10, 159-162. https://doi.org/10.1103/PhysRevLett.10.159

[3] Kanamori, J. (1963) Electron Correlation and Ferromagnetism of Transition Metals. Progress of Theoretical Physics, 30, 275-289. https://doi.org/10.1143/PTP.30.275

[4] Anderson, P.W. (1961) Localized Magnetic States in Metals. Physical Review, 124, 41-53. https://doi.org/10.1103/PhysRev.124.41

[5] Shubin, S.P. and Wonsowsky, S.V. (1934) On the Electron Theory of Metals. Proceedings of the Royal Society A, 145, 159-172.

https://doi.org/10.1098/rspa.1934.0089

[6] Karpenko, B.V., Dyakin, V.V. and Budrina, G.L. (1986) Two Electrons in the Hubbard Model. Physics of Metals and Metallography, 61, 702-706.

[7] Mattis, D. (1986) The Few-Body Problems on a Lattice. Reviews of Modern Physics, 58, 370-379. https://doi.org/10.1103/RevModPhys.58.361

[8] Lieb, E. (1989) Two Theorems on the Hubbard Model. Physical Review Letters, 62, 1201-1204. https://doi.org/10.1103/PhysRevLett.62.1201

[9] Tsvelick, A.M. and Wiegman, P.B. (1983) Exact Results in the Theory of Magnetic Alloys. Advances in Physics, 32, 453-713. https://doi.org/10.1080/00018738300101581

[10] Izyumov, Yu.A. and Skryabin, Yu.N. (1988) Statistical Mechanics of Magnetically Ordered Systems. Nauka, Moscow. (In Russian)

[11] Tashpulatov, S.M. (2014) Spectral Properties of Three-Electron Systems in the Hubbard Model. Theoretical and Mathematical Physics, 179, 712-728. https://doi.org/10.1007/s11232-014-0173-y

[12] Tashpulatov, S.M. (2016) Spectra of the Energy Operator of Four-Electron Systems in the Triplete State in the Hubbard Model. Journal of Physics: Conference Series, 697, Article ID: 012025. https://doi.org/10.1088/1742-6596/697/1/012025

[13] Tashpulatov, S.M. (2017) The Structure of Essential Spectra and Discrete Spectrum of Four-Electron Systems in the Hubbard Model in a Singlet State. Lobachevskii Journal of Mathematics, 38, 530-541. https://doi.org/10.1134/S1995080217030246

[14] Tashpulatov, S.M. (2019) The Spectrum of the Energy Operator in Three-Electron Systems with an Impurity in the Hubbard Model. The Second Doublet State. Modern Problems of Mathematics, and Physics. Moscow, 65, 109-123. https://doi.org/10.22363/2413-3639-2019-65-1-109-123

[15] Tashpulatov, S.M. (2021) The Structure of Essential Spectra and Discrete Spectrum of Three-Electron Systems in the Impurity Hubbard Model. Quartet State. Journal of Applied Mathematics and Physics, 9, 1391-1421.

https://doi.org/10.4236/jamp.2021.96094 
[16] Reed, M. and Simon, B. (1972) Methods of Modern Mathematical Physics, Vol. 1, Functional Analysis. Academic Press, New York.

[17] Reed, M. and Simon, B. (1982) Methods of Modern Mathematical Physics, Vol. 4, Operator Analysis. Academic Press, New York. 